\title{
Caracterización edáfica y dasométrica de una plantación de Eucalyptus globulus Labill y propuesta de manejo en la zona estepa espinosa Montano Bajo, Riobamba, Ecuador
}

\section{(Soil and dasometric characterization of a Eucalyptus globulus Labill plantation and management proposal in the lower montane thorny steppe zone, Riobamba, Ecuador)}

\author{
Miguel Guallpa ${ }^{1}$, Sonia Rosero ${ }^{1}$, María Samaniego ${ }^{1}$, Eduardo Cevallos ${ }^{1}$
}

\begin{abstract}
Resumen:
Existe escasa información sobre el crecimiento y rendimiento de los árboles de la plantación de Eucalyptus globulus Labill en la hacienda Tunshi-ESPOCH en condiciones variables de fisiografía, propiedades edáficas y manejo; por esta razón se decidió evaluar su masa forestal. Se instalaron parcelas circulares de $12.62 \mathrm{~m}$ de radio a una intensidad de 5-6\% ha ${ }^{-1}$. Mediante el muestreo sistemático no alineado para el levantamiento de información dasométrica y la excavación de tres calicatas rodal ${ }^{-1}$, aplicando el muestreo estratificado para las variables del sitio. La aplicación de estimadores estadísticos, determinó que a una altitud de $2755 \mathrm{msnm}$ el volumen total promedio árbol ${ }^{-1}$ estimado de $2,32 \mathrm{~m}^{3}$ en comparación con el $0,25 \mathrm{~m}^{3}$ a una altitud de $2929 \mathrm{msnm}$. Se definieron dos áreas una de conservación y otra de aprovechamiento forestal. Existe asociaciones significativas entre el volumen total unitario de $E$. globulus frente a la altitud, al contenido de materia orgánica, a la capacidad de intercambio catiónico a nivel superficial y el nivel de boro a nivel subyacente. Generándose una estrategia de manejo para cada área delimitada.
\end{abstract}

Palabras clave: suelos; dasometría; plantación forestal; productividad; Eucalyptus globulus.

\begin{abstract}
:
There is limited information on the growth and yield of the Tasmanian blue gum plantation in Tunshi-ESPOCH homestead in varying physiographic conditions, soil properties and management; for this reason it was decided to assess its forest mass. Circular plots were installed with $12.62 \mathrm{~m}$ radius at an intensity of $5-6 \% \mathrm{ha}^{-1}$. Through systematic unaligned sampling for lifting dasometric information and digging three pits stand $^{-1}$, using stratified sampling for variables of the place. The application of statistical estimators, determined at an altitude of $2755 \mathrm{amsl}$, a total average tree volume estimated ${ }^{-1}$ of $2.32 \mathrm{~m}^{3}$ compared with $0.25 \mathrm{~m}^{3}$ at an altitude of 2929 amsl. Two areas of conservation and other forest use were defined. There are significant associations between total unit volume of $T$. blue against altitude, organic matter content, cationic exchange capacity at surface level and level of boron underlying level, generating a management strategy for each defined area.
\end{abstract}

Keywords: Soils; dasometric; forest plantation; productivity; Tasmanian blue gum.

\footnotetext{
${ }^{1}$ Escuela Superior Politécnica de Chimborazo, Riobamba - Ecuador ( \{miguel.guallpa, s_rosero, maria.samaniego, e_cevallos\} @espoch.edu.ec )
} 


\section{Introducción}

El Eucalyptus globulus Labill actualmente está presente en más de 90 países, la mayoría en zonas tropicales y subtropicales: Nueva Zelanda, Chile, Argentina, Brasil, Uruguay, Sudáfrica, la Península Ibérica y Estados Unidos (ENCE, 2009). Existen aproximadamente 5 millones de hectáreas que corresponden a E. globulus Labill (Soto, 2011).

En Ecuador el 34,7 \% de la superficie nacional está cubierta por bosques, el 98,5\% son bosques naturales, las plantaciones no superan el 1,5\% restante del patrimonio forestal (Grijalva et al., 2012). Durante el período 2007-2010, la madera autorizada para aprovechamiento con el 58,5\% que correspondió a plantaciones forestales (Palacios \& Quiroz, 2012).

Para el período 2008 - 2009, se autorizó aprovechar 580,2 miles de $\mathrm{m}^{3}$ provenientes de las provincias de Pichincha, Cotopaxi y Chimborazo, la cual ocupa el primer lugar con el 19,43\% del volumen total de madera autorizada y aprovechada del país (MAE, 2010).

El eucalipto es una especie de alta capacidad productiva que prospera en terrenos forestales degradados o inutilizados, se desarrolla en condiciones edafoclimáticas diversas (ENCE, 2009). En la región sierra, es priorizada para la reforestación con fines comerciales debido a su adaptabilidad, rápido crecimiento y rentabilidad (MAGAP. 2015). La inadecuada aplicación de técnicas al establecimiento y manejo influye en el incremento medio anual con valores de 10 a 12 $\mathrm{m}^{3} \mathrm{ha}^{-1}$ año ${ }^{-1}$ el nivel más bajo del crecimiento proyectado, en contraste con los $32 \mathrm{~m}^{3} \mathrm{ha}^{-1}$ año ${ }^{-1}$, aplicando el manejo en plantaciones de la provincia de Osorno-Chile (Geldres \& Schlatter, 2004).

El limitado crecimiento y calidad de los árboles de eucalipto a nivel de sitio y micrositio en el sector de Tunshi, en condiciones adversas, y la ausencia de labores silviculturales, motivó evaluar su crecimiento y productividad, para disponer de su categorización como áreas de protección o producción. Este hecho se justifica por aspectos: tamaño de la propiedad, cuidados silviculturales inexistentes (Diaz Balteiro et al., 2008). En este contexto es fundamental ejecutar la administración, el manejo del bosque, a fin de obtener una óptima y continua productividad del sitio, con las condiciones tecnológicas y socio-económicas prevalecientes (Hernández J. , 2014).

A partir del inventario del bosque plantado, se levantó la información considerando los parámetros edafoclimáticos y dasométricos de la especie (Nájera \& Hernández, 2008). El objetivo del presente estudio fue tipificar los suelos y evaluar la productividad de cada rodal. Se comprobó asociaciones significativas entre el volumen total árbol ${ }^{-1}$ frente a ciertos parámetros del sitio. Con ello se dispone de instrumentos prácticos en la aplicación de técnicas silvícolas, para mejorar su productividad, lo que influye en la rentabilidad final (Sharma et al., 2001). 


\section{Materiales y Métodos}

\section{1 Área de estudio}

La presente investigación se realizó en la plantación de E. globulus Labill de 35 años de edad, ubicada en la Estación Experimental Tunshi de la Escuela Superior Politécnica de Chimborazo, Tunshi Grande, parroquia Licto, al suroeste del cantón Riobamba, provincia de ChimborazoEcuador, a $12 \mathrm{~km}$ de la ciudad capital. Geográficamente la masa forestal se inserta dentro de los puntos de referencia: UTM Zona 17S Datum WGS $84 X_{1}=763609 ; Y_{1}=9806880, X_{2}=764973 ; Y_{2}$ $=9806791, X_{3}=764973 ; Y_{3}=9805248$ y $X_{4}=763606 ; Y_{4}=9805251$. Se encuentra ubicada a una altitud que va desde 2755 - $2929 \mathrm{msnm}$. La precipitación anual es 635,4 mm, la temperatura media es de $14,4^{\circ} \mathrm{C}$ y hay una humedad relativa media de 85 \%. Datos de la Estación Guaslan; serie 1990-2012; MAGAP. La plantación tiene una superficie neta de 37,62 ha, un área sin vegetación de 1,38 has dando un total de 39,00 ha y se categoriza dentro de la formación: Matorral Húmedo Montano de los Andes del Norte y Centro (Sierra et al., 1999).

El área de plantación presentó las siguientes características fisiográficas (Tabla 1).

Tabla 1. Principales características fisiográficas y del suelo en el área de estudio.

\begin{tabular}{|c|c|c|c|c|}
\hline Rodal & $\begin{array}{l}\text { Altitud } \\
\text { (msnm) }\end{array}$ & $\begin{array}{l}\text { Pendiente } \\
(\%)\end{array}$ & $\begin{array}{l}\text { Nivel de pedregosidad interna } \\
(\%)\end{array}$ & $\begin{array}{l}\text { Nivel de drenaje interno } \\
\qquad(\mathrm{cm} / \mathrm{hr})\end{array}$ \\
\hline 1 & 2787 & 20 & No pedregoso & Drenaje moderado \\
\hline 2 & 2861 & 55 & No pedregoso & Drenaje moderadamente lento \\
\hline 3 & 2929 & 55 & No pedregoso & Drenaje moderadamente lento \\
\hline 4 & 2894 & 58 & Moderadamente pedregoso & Drenaje moderadamente lento \\
\hline 5 & 2793 & 57 & No pedregoso & Drenaje moderadamente lento \\
\hline 6 & 2774 & 50 & No pedregoso & Drenaje moderado \\
\hline 7 & 2755 & 18 & Moderadamente pedregoso & Drenaje moderado \\
\hline
\end{tabular}

No pedregosos $=<5 \%$; Moderadamente pedregoso 5-20\%; Drenaje moderadamente lento $=0,51-2,00$ $\mathrm{cm} \mathrm{hr}^{-1}$; Drenaje moderado $=2,00-6,30 \mathrm{~cm} \mathrm{hr}^{-1}$

\subsection{Métodos}

Los muestreos de los componentes: edáfico y arbóreo tuvieon lugar en el último semestre del 2014.

\subsection{Características edáficas}

En el levantamiento de las propiedades edáficas, se aplicó el muestreo estratificado con la excavación de 3 calicatas ( $1 \mathrm{~m}$ largo x $1 \mathrm{~m}$ ancho y $1 \mathrm{~m}$ de profundidad) por rodal, con el objeto de recopilar una muestra de suelo profundidad ${ }^{-1}$, entre 0-30 y de 30-100 cm, además se identificó la 
profundidad efectiva del suelo $(\mathrm{m})$ al comprobarlo con una cinta métrica. La cobertura herbácea $(\%)$, se determinó con el método del cuadrante $\left(1 \mathrm{~m}^{2}\right)$ con tres repeticiones, ubicadas en sus cuatro puntos cardinales, identificando las especies existentes; se estimó el área ocupada y a la vez el grado de erosión por apreciación (Kaplán et al., 2011).

La caracterización física y química de los suelos: color en seco y en húmedo (Munsell), textura (hidrómetro), estructura (estabilidad de los agregados), la densidad aparente en $\mathrm{g} \mathrm{cm}^{3-1}$ (cilindro graduado), \% materia orgánica (ignición), potencial de hidrógeno (potenciométrico), el nitrógeno $\left(\mathrm{NH} 4 ; \mathrm{mg} \mathrm{L}^{-1}\right)$, fósforo $\left(\mathrm{P} ; \mathrm{mg} \mathrm{L}^{-1}\right)$, potasio $\left(\mathrm{K} ; \mathrm{Meq} 100 \mathrm{~g}^{-1}\right)$, con solución extractora de Olsen, la capacidad de intercambio catiónico en Meq $100 \mathrm{~g}^{-1}$ (Bouyoucos modificado y materia orgánica), realizados en el laboratorio de suelos de la Facultad de Recursos Naturales-ESPOCH y el boro ppm (curcumina) en el Instituto Nacional de Investigaciones Agropecuarias (INIAP, 2014).

\subsection{Cobertura forestal}

Se instalaron parcelas circulares con un radio de 12,62 m y separadas entre ellas a 75 y $100 \mathrm{~m}$, con el diseño de muestreo sistemático no alineado (FAO, 2011); al considerar la topografía y exposición de cada rodal, se aplicó una intensidad de muestreo del 5-6 \% ha ${ }^{-1}$.

En las parcelas se observaron los árboles: brinzales, latizales y se contaron los árboles fustales $(\varnothing>$ a $10 \mathrm{~cm}$ ), se midió su diámetro (forcípula Haglof Mantax) y altura total $(\mathrm{HT} ; \mathrm{m})$ con el clinómetro Suunto PM-5/1520PC. El cálculo del área basal con la fórmula (Ecuador forestal, 2010)

$$
A B=\frac{\pi \times D^{2}}{4}
$$

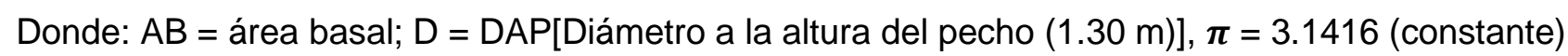

La ecuación utilizada de volumen total del árbol en pie (Ecuador forestal, 2010).

$$
V=\frac{3.1416 \times(D A P)^{2} \times h \times f f}{4}
$$

Donde: $\mathrm{V}=$ volumen total del árbol $\left(\mathrm{m}^{3}\right), \quad \mathrm{DAP}=$ Diámetro del árbol a la altura del pecho $(\mathrm{m}) ; \mathrm{h}=$ Altura comercial o total del árbol $(\mathrm{m}), \mathrm{ff}=$ factor de forma $=0.7$

La densidad de cobertura (D) mediante la ecuación (Alvarado, 2013).

$$
D=\frac{A B}{H T} \times 100
$$

Donde: $\mathrm{D}=$ Densidad de cobertura en $\%, \mathrm{AB}=$ Área basal $\left(\mathrm{m}^{2} \mathrm{ha}^{-1}\right), \mathrm{HT}=$ Altura total promedio de los árboles del rodal (m). 


\subsection{Análisis estadístico}

Mediante el cálculo de estimadores estadísticos al procesar los parámetros: físico-químicos del suelo, variables dasométricas. Formación de conglomerados y la prueba de Spearman Roms. En los análisis se utilizó la hoja de cálculo de Excel y los softwares: SPSS Statistics 18 e Infostat.

\section{Resultados}

\subsection{Variables edáficas}

En la mayoría de los suelos en estudio, la profundidad efectiva del suelo es delgada $(25-50 \mathrm{~cm})$ a excepción del rodal 7 que presentó una profundidad moderada $(75-100 \mathrm{~cm})$, con una cobertura herbácea variable desde 31,67-75\% en los rodales 1 y 6 respectivamente. Presenta una erosión moderada en la superficie de los rodales 5,6 y 7 hasta severa en las áreas de los rodales 1, 2, 3, 4.

A las dos profundidades de $0-30 \mathrm{~cm}$ y $30-100 \mathrm{~cm}$ se aprecia similar condición textural franca arenosa en los suelos de la mayoría de rodales, a excepción del rodal 4, donde se detectó una textura arena franca con una estructura masiva (tabla 2).

En la Tabla 2, a las profundidades de 0-30 y 30-100 cm, el color en seco y húmedo va desde 10YR 4/2 gris muy oscuro en el suelo del rodal 3 hasta 10YR 6/3 pardo pálido en el caso del rodal 4 en seco y un color 10YR 5/2 pardo grisáceo en los rodales 4 y 5 en húmedo.

La densidad aparente de los suelos a una profundidad de $0-30 \mathrm{~cm}$ es de $1,60 \mathrm{~g} \mathrm{~cm}^{3-1}$ en los rodales 3,6 a menor en el rodal 4 con $1,52 \mathrm{~g} \mathrm{~cm}^{3-1}$ y de $30-100 \mathrm{~cm}$ el suelo del rodal 2 presentó una mayor densidad con $1,62 \mathrm{~g} \mathrm{~cm}^{3-1}$ y la menor densidad aparente con $1,53 \mathrm{~g} \mathrm{~cm}^{3-1}$ corresponde al suelo del rodal 6 (Tabla 2).

A profundidades de 0-30 y $30-100 \mathrm{~cm}$, al determinar las propiedades químicas para el contenido de materia orgánica cuyos valores oscilan entre 1,10 a 2,77 \% ubicándose en un nivel bajo $(<3$ \%). La capacidad de intercambio catiónico con un nivel bajo (<12 Meq 100 $\mathrm{g}^{-1}$ ) (tablas 3 y 4 ).

Al determinar las propiedades químicas del suelo de 0-30 y 30-100 cm se encontró que el potencial de hidrógeno $(\mathrm{pH})$ fluctúa entre ligeramente alcalino hasta alcalino; los contenidos nutricionales: el nitrógeno bajo $\left(<30.00 \mathrm{mg} \mathrm{L}^{-1}\right)$; el fósforo alto $\left(>30,00 \mathrm{mg} \mathrm{L}^{-1}\right)$; el potasio alto (> $\left.0,64 \mathrm{Meq}^{100 \mathrm{~g}^{-1}}\right)$, para el boro bajo (<0.90 ppm) (tablas 3 y 4$)$. 
Tabla 2. Principales características físicas de 0-30 y de 30-100 cm de profundidad en el área de estudio.

\begin{tabular}{|c|c|c|c|c|c|c|c|c|c|}
\hline $\begin{array}{l}\text { ग } \\
\text { Oे } \\
\stackrel{0}{0}\end{array}$ & $\begin{array}{l}\text { Prof. } \\
\text { efectiva } \\
\text { del suelo } \\
\text { (cm) }\end{array}$ & $\begin{array}{c}\text { Cobertura } \\
\text { herbácea } \\
\text { del suelo } \\
(\%)\end{array}$ & $\begin{array}{l}\text { Grado de } \\
\text { erosión }\end{array}$ & $\begin{array}{l}\text { Prof } \\
\text { (cm) }\end{array}$ & $\begin{array}{l}\text { Color en } \\
\text { seco }\end{array}$ & $\begin{array}{l}\text { Color en } \\
\text { húmedo }\end{array}$ & Textura & Estructura & $\begin{array}{l}\text { Densidad } \\
\text { aparente } \\
\left(\mathrm{gcm}^{3-1}\right)\end{array}$ \\
\hline \multirow{2}{*}{1} & \multirow{2}{*}{ Delgada } & \multirow{2}{*}{31,67} & \multirow{2}{*}{ Severa } & $0-30$ & 10YR 6/2 & 10YR 3/2 & $\mathrm{Fa}$ & Masiva & 1,59 \\
\hline & & & & $30-100$ & 10YR 3/3 & 10YR 3/2 & $\mathrm{Fa}$ & Masiva & 1,61 \\
\hline \multirow{2}{*}{2} & \multirow{2}{*}{ Delgada } & \multirow{2}{*}{50,00} & \multirow{2}{*}{ Severa } & $0-30$ & 10YR $3 / 3$ & 10YR 3/2 & $\mathrm{Fa}$ & Masiva & 1,54 \\
\hline & & & & $30-100$ & 10YR 6/2 & 10YR 3/2 & $\mathrm{Fa}$ & Masiva & 1,62 \\
\hline \multirow{2}{*}{3} & \multirow{2}{*}{ Delgada } & \multirow{2}{*}{43,33} & \multirow{2}{*}{ Severa } & $0-30$ & 10YR 4/2 & 10YR 4/2 & $\mathrm{aF}$ & Masiva & 1,60 \\
\hline & & & & $30-100$ & 10YR 6/2 & 10YR 3/2 & $\mathrm{Fa}$ & Masiva & 1,54 \\
\hline \multirow{2}{*}{4} & \multirow{2}{*}{ Delgada } & \multirow{2}{*}{48,33} & \multirow{2}{*}{ Severa } & $0-30$ & 10YR 6/3 & 10YR 5/2 & $\mathrm{Fa}$ & Masiva & 1,52 \\
\hline & & & & $30-100$ & 10YR 6/3 & 10YR 5/2 & $\mathrm{Fa}$ & Masiva & 1,61 \\
\hline \multirow{2}{*}{5} & \multirow{2}{*}{ Delgada } & \multirow{2}{*}{58,33} & \multirow{2}{*}{ Moderada } & $0-30$ & 10YR 6/1 & 10YR 5/2 & $\mathrm{Fa}$ & Masiva & 1,57 \\
\hline & & & & $30-100$ & 10YR 3/3 & 10YR 5/2 & $\mathrm{Fa}$ & Masiva & 1,57 \\
\hline \multirow{2}{*}{6} & \multirow{2}{*}{ Delgada } & \multirow{2}{*}{75,00} & \multirow{2}{*}{ Moderada } & $0-30$ & 10YR 3/3 & 10YR 3/2 & $\mathrm{Fa}$ & Masiva & 1,60 \\
\hline & & & & $30-100$ & 10YR 3/3 & 10YR 3/2 & $\mathrm{Fa}$ & Masiva & 1,53 \\
\hline \multirow{2}{*}{7} & \multirow{2}{*}{$\begin{array}{l}\text { Modera- } \\
\text { damente } \\
\text { profundo }\end{array}$} & \multirow{2}{*}{35,00} & \multirow{2}{*}{ Moderada } & $0-30$ & 10YR 3/3 & 10YR 3/2 & $\mathrm{Fa}$ & Masiva & 1,58 \\
\hline & & & & $30-100$ & 10YR $6 / 1$ & 10YR 5/2 & $\mathrm{Fa}$ & Masiva & 1,57 \\
\hline
\end{tabular}

Delgada $=25-50 \mathrm{~cm}$, Ligeramente profundo $=50-75 \mathrm{~cm}$; Moderadamente profundo $=75-100 \mathrm{~cm} ; 10 \mathrm{YR} 4 / 2$ (Gris muy oscuro); 10YR 3/2 (Pardo grisáceo muy oscuro); 10YR 5/2 (Pardo grisáceo); 10YR 6/2 (Pardo grisáceo claro); 10YR 3/3 (Pardo oscuro); 10YR 6/1 (Pardo); 10YR 6/3(Pardo pálido); Fa= Franca arenosa; aF=arena franca

Tabla 3. Aplicación de estimadores estadísticos a los parámetros físicos y químicos de 0-30 cm de profundidad.

\begin{tabular}{|l|c|c|c|c|c|c|c|}
\hline Rodales & $\mathbf{M O}$ & $\mathbf{p H}$ & $\mathbf{N H 4}$ & $\mathbf{P}$ & $\mathbf{K}$ & $\mathbf{C l C}$ & $\mathbf{B}$ \\
\hline & $(\%)$ & & $\left(\mathrm{mg} \mathrm{L}^{-1}\right)$ & $\left(\mathrm{mg} \mathrm{L}^{-1}\right)$ & $\left(\mathrm{Meq100g}^{-1}\right)$ & $\left({\left.\mathrm{Meq} 100 \mathrm{~g}^{-1}\right)}^{\mathrm{Ppm}}\right.$ \\
\hline 1 & 1,10 & 8,57 & 3,18 & 32,20 & 0,67 & 3,23 & 0,30 \\
\hline 2 & 1,90 & 8,93 & 3,09 & 50,83 & 1,66 & 4,60 & 0,37 \\
\hline 4 & 2,20 & 8,30 & 3,41 & 39,10 & 0,42 & 5,17 & 0,33 \\
\hline 5 & 2,77 & 8,93 & 3,82 & 41,13 & 1,56 & 6,13 & 0,33 \\
\hline 6 & 1,47 & 8,67 & 4,03 & 41,73 & 1,49 & 4,23 & 0,33 \\
\hline 7 & 1,53 & 8,77 & 3,63 & 39,17 & 0,85 & 4,33 & 0,40 \\
\hline Media & 1,17 & 7,43 & 4,40 & 54,83 & 0,70 & 3,70 & 0,43 \\
\hline Desv. Est. & 1,73 & 8,51 & 3,65 & 42,71 & 1,05 & 4,49 & 0,36 \\
\hline Coef. Varia. & 0,60 & 0,53 & 0,47 & 7,66 & 0,51 & 0,95 & 0,05 \\
\hline Error. Muest & 30,85 & 6,17 & 12,87 & 17,94 & 48,20 & 21,27 & 12,88 \\
\hline
\end{tabular}

Codificación utilizada para las tablas 3 y 4 . Materia orgánica (MO), Potencial de hidrógeno $(\mathrm{pH})$, Nitrógeno $\left(\mathrm{NH}_{4}\right)$, Fósforo $(\mathrm{F})$, Potasio $(\mathrm{K})$, Capacidad de intercambio catiónico (CIC) y Boro (B). 
Tabla 4. Aplicación de estimadores estadísticos a los parámetros físicos y químicos de 30-100 cm de profundidad.

\begin{tabular}{|l|c|c|c|c|c|c|c|}
\hline Rodales & $\mathbf{M O}$ & $\mathbf{p H}$ & $\mathbf{N H 4}$ & $\mathbf{P}$ & $\mathbf{K}$ & $\mathbf{C I C}$ & $\mathbf{B}$ \\
\hline & $(\%)$ & & $\left(\mathrm{mg} \mathrm{L}^{-1}\right)$ & $\left(\mathrm{mg} \mathrm{L}^{-1}\right)$ & $\left(\mathrm{Meq} \mathrm{100g}^{-1}\right)$ & $\left(\mathrm{Meq} \mathrm{100g}^{-1}\right)$ & $\mathrm{Ppm}$ \\
\hline 1 & 1,10 & 9,30 & 3,25 & 51,40 & 1,28 & 2,87 & 0,40 \\
\hline 2 & 2,77 & 9,50 & 2,98 & 47,70 & 3,03 & 6,13 & 0,30 \\
\hline 3 & 2,03 & 8,53 & 3,27 & 38,70 & 0,51 & 4,53 & 0,43 \\
\hline 4 & 2,73 & 9,50 & 3,65 & 48,47 & 2,13 & 6,10 & 0,40 \\
\hline 5 & 1,27 & 8,53 & 4,81 & 44,33 & 1,93 & 3,20 & 0,27 \\
\hline 6 & 2,43 & 9,37 & 3,18 & 51,90 & 2,00 & 6,57 & 0,30 \\
\hline 7 & 1,30 & 9,03 & 4,02 & 58,20 & 1,68 & 3,53 & 0,23 \\
\hline Media & 1,95 & 9,11 & 3,59 & 48,67 & 1,79 & 4,70 & 0,33 \\
\hline Desv. Est. & 0,72 & 0,42 & 0,64 & 6,16 & 0,78 & 1,55 & 0,08 \\
\hline Coef. Varia. & 37,10 & 4,65 & 17,78 & 12,66 & 43,40 & 33,04 & 23,09 \\
\hline Error. Muest. & 33,17 & 4,16 & 15,89 & 11,32 & 38,80 & 29,53 & 20,64 \\
\hline
\end{tabular}

\subsection{Prácticas empleadas en el establecimiento y manejo de la plantación}

No se realizaron fertilización, coronamiento y otras actividades de manejo: raleos, manejo de rebrotes, solo existe un reporte del monitoreo parcial de la plantación para aprovechar los árboles en los perímetros de los rodales 1, 5, 6, y 7. Se identificaron también brinzales y latizales defectuosos como efectos de las intervenciones antrópicas de pobladores del sector.

\subsection{Variables dasométricas}

El inventario demuestra que la masa arbórea está formada por una sola especie en diferentes etapas de desarrollo: los brinzales y latizales resultantes del efecto de actividades antrópicas.

Tabla 5. Variables dasométricas con la estimación estadística.

\begin{tabular}{|l|c|c|c|c|c|c|c|}
\hline Rodal & DAP & $\mathbf{H T}$ & $\mathbf{A B P}$ & $\mathbf{N}$ & $\mathbf{A B ~ h a}^{-1}$ & $\mathbf{D}$ & VTPárbol $^{-1}$ \\
\hline 1 & $\mathrm{~cm}$ & $\mathbf{M}$ & $\mathrm{m}^{2}$ & & $\mathrm{~m}^{2}$ & $\%$ & $\mathrm{~m}^{3}$ \\
\hline 2 & 21,00 & 21,63 & 0,039 & 472,94 & 18,39 & 85,04 & 0,68 \\
\hline 3 & 15,83 & 17,11 & 0,022 & 640,00 & 13,77 & 80,50 & 0,30 \\
\hline 4 & 14,88 & 14,66 & 0,019 & 320,00 & 6,08 & 41,50 & 0,25 \\
\hline 5 & 14,47 & 13,88 & 0,019 & 460,00 & 8,51 & 61,35 & 0,23 \\
\hline 6 & 29,90 & 30,84 & 0,079 & 460,00 & 36,16 & 117,25 & 1,88 \\
\hline 7 & 28,62 & 26,03 & 0,076 & 375,00 & 28,63 & 109,96 & 1,84 \\
\hline Media & 37,52 & 24,46 & 0,122 & 360,00 & 43,96 & 179,72 & 2,32 \\
\hline Dato ha & 23,17 & 21,23 & 0,054 & 441,13 & 22,21 & 96,47 & 1,07 \\
\hline Desv. Est. & & & & 441,13 & 22,21 & 96,47 & 472,09 \\
\hline Coef. Varia. & 3,98 & 6,33 & 0,040 & 105,59 & 14,39 & 45,06 & 0,91 \\
\hline Error. Muest. & 34,64 & 29,81 & 74,321 & 23,94 & 64,77 & 46,71 & 84,79 \\
\hline
\end{tabular}

Diámetro a la altura del pecho (DAP), Altura total (HT), Área basal promedio árbol ${ }^{-1}(A B P)$,

Número de árboles fustales (N), Área basal ha ${ }^{-1}\left(A B h^{-1}\right)$, Densidad de cobertura (D),

Volumen total promedio árbol ${ }^{-1}$ (VTPárbol $\left.{ }^{-1}\right)$. 
En estado fustal con un DAP > a $10 \mathrm{~cm}$ se contabilizaron 441,13 árboles ha ${ }^{-1}$; estos tienen un DAP promedio de $23,17 \mathrm{~cm}$, con una altura total de $21,23 \mathrm{~m}$.

Como se muestra en la Tabla 5, la media aritmética del área basal de los siete rodales para el $E$. globulus Labill es de 0,54 $\mathrm{m}^{2}$ árbol ${ }^{-1}$, con 441,13 árboles, se estima $22,21 \mathrm{~m}^{2} \mathrm{ha}^{-1}$ de área basal y una densidad de cobertura del $96,47 \%$ de área basal con un volumen total de $472,09 \mathrm{~m}^{3} \mathrm{ha}^{-1}$.

\subsection{Agrupamiento de subáreas de manejo para la conservación y aprovechamiento.}

Con base en las variables dasométricas; área basal ha $^{-1}$, la densidad de cobertura en \% de los 7 rodales y el Volumen total promedio árbol ${ }^{-1}$, utilizando el análisis de conglomerados, se construyó un dendrograma que muestra la asociación entre rodales, con dos grupos claramente identificados, el primero conformado por los rodales 1, 2, 3 y 4 y el segundo por los rodales 5,6 y 7 (Figura 1).

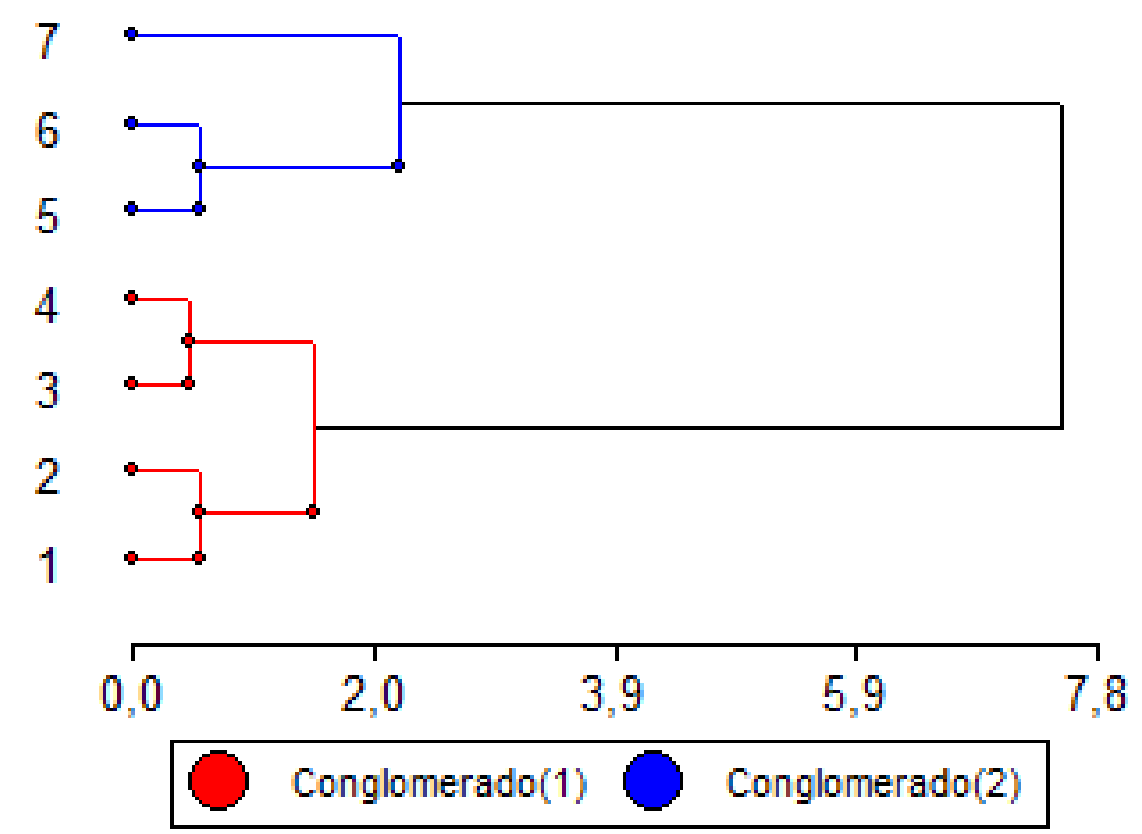

Figura 1. Dendrograma resultante del análisis de conglomerados (método de ward, distancia Euclídea) para los 7 rodales en estudio.

\subsection{Análisis de la varianza multivariado}

EI MANOVA basado en las variables de cada grupo y la prueba de Roy, mostraron diferencias altamente significativas ( $p<0,0082$, Tabla 6$)$. 
Tabla 6. Análisis de la varianza (Roy)

\begin{tabular}{|c|r|r|r|r|l|}
\hline FV. & \multicolumn{1}{|c|}{ Estadístico } & F & gl (num) & gl (den) & \multicolumn{1}{c|}{ P } \\
\hline Conglomerados & 33,82 & 33,82 & 3 & 3 & 0,0082 \\
\hline
\end{tabular}

Este resultado (Tabla 6) nos permite valorar dos estrategias de manejo forestal con base a los estudios dasométricos, una para cada conglomerado (áreas de conservación y aprovechamiento).

\subsection{Asociación entre las variables volumen total árbol ${ }^{-1} \mathrm{y}$ del sitio.}

Al aplicar el método de Spearman Roms entre el volumen total del árbol ${ }^{-1}$ y las variables independientes: altitud, contenido de materia orgánica, capacidad de intercambio catiónico entre 0-30 cm y para el boro desde $30-100 \mathrm{~cm}$ de profundidad, muestra cuatro correlaciones negativas y significativas $(p<0,05)$, en la Tabla 7 .

Tabla 7. Correlación no paramétrica de Spearman Roms

\begin{tabular}{|l|l|r|r|r|r|}
\hline \multicolumn{2}{|c|}{} & Altitud & \multicolumn{1}{c|}{ MO } & \multicolumn{1}{c|}{ CIC } & \multicolumn{1}{c|}{ B } \\
\hline & & $(\mathrm{msnm})$ & \multicolumn{1}{c|}{$(\%)$} & $\left(\mathrm{Meq} 100 \mathrm{~g}^{-1}\right)$ & \multicolumn{1}{c|}{$(\mathrm{ppm})$} \\
\hline \multirow{3}{*}{$\begin{array}{l}\text { Volumen total del } \\
\text { árbol }^{-1}\end{array}$} & Coeficiente de correlación &,$- 857^{*}$ &,$- 786^{*}$ &,$- 786^{*}$ &,$- 855^{*}$ \\
\cline { 2 - 7 } & Sig. (bilateral) &, 014 &, 036 &, 036 &, 014 \\
\cline { 2 - 7 } & $\mathrm{N}$ & 7 & 7 & 7 & 7 \\
\hline
\end{tabular}

*La correlación es significativa al nivel 0,05 (bilateral)

\section{Discusión}

\subsection{Parámetros físicos del suelo}

La profundidad efectiva del suelo en Tunshi es delgada a excepción de un lote que es moderadamente profundo, esta limitante influye en la capacidad de anclaje y tienen mayor susceptibilidad de caer los árboles (Trujillo E. , 2013).

La cobertura herbácea del suelo se sitúa entre abierta y cerrada formada por Stipa ichu, Pennisetum clandestinum, Cortaderia jubata, Baccharis latifolia, esporádicamente Niphidium albopinctattissimum y Dodonea viscosa, plantas tolerantes a una poca profundidad del suelo (Aguirre \& Aguirre, 2010). Complementándose con la hojarasca en proceso de descomposición.

El eucalipto requiere un suelo bien drenado para su óptimo desarrollo (Trujillo E., 2013); la investigación revela un drenaje moderadamente lento a moderado con deficiente drenaje; los colores grises indican mal drenaje (Jaramillo, 2002), coincidiendo los resultados en estado seco y húmedo, varían de pardo grisáceo a gris oscuro a nivel superior y subyacente. Requiere suelos 
de textura franca arcillosa, franca arenosa o arenosa (Trujillo E., 2013); lo cual concuerda con la textura observada en nuestra área de estudio.

Los suelos con estructura masiva tienen normalmente una consistencia más fuerte y compacta en cuanto a ruptura (FAO, 2009); similar a la estructura masiva identificada en los suelos del área en estudio limitando el desarrollo del sistema radicular, que contrasta con las estructuras: bloque subangulares a $18 \mathrm{~cm}$, tipo columnar entre $18-50 \mathrm{~cm}$ reportada en plantaciones E. globulus Labill, provincia de Pichincha (Ramírez, 2012). La densidad aparente de los suelos de textura franca arenosa, varía: 1,53-1,62 $\mathrm{g} \mathrm{cm}^{3-1}$ a excepción del suelo del rodal 3 a nivel superficial de textura arena franca con valores iguales y menores a $1,60 \mathrm{~g} \mathrm{~cm}^{3-1}$, insertándose en el criterio ideal para el crecimiento radicular (Bravo C. , 2014), valores superiores al $1,39 \mathrm{~g} \mathrm{~cm}^{3-1}$, reportado en suelos de laderas reforestadas (Hernández et al., 2008).

Comparativamente los valores determinados de contenido de materia orgánica son bajos frente $15,09 \%$ obtenido en suelos de Asturias (Afif et al., 2014) y similares a los reportados para el horizonte superficial, en el E. globulus que entre 1 y $3 \%$; en los suelos más pobres es menor (Díaz Hernández \& Sánchez Marañón, 1988-b).

\subsection{Parámetros químicos del suelo}

Los suelos de la localidad de Tunshi tiene un pH alcalino, influenciado por el nivel de precipitación en la zona (INPOFOS, 1997); resultado que no concuerda con los niveles de acidez valorado a $10 \mathrm{~cm}$ de profundidad, en rodales de eucalipto en el SO español (Domingo-Santos et al., 2009).

El contenido de nitrógeno es 54,5 veces menor al valor de $200 \mathrm{mg} \mathrm{L}^{-1}$ reportado en plantaciones de E. globulus en la Cuenca Media del Río Paute (Mejía \& Moscoso, 2010); 779,94 veces menor al reporte de $2800 \mathrm{mg} \mathrm{L}^{-1}$ en suelos de Asturias (Afif et al., 2014), el nivel bajo de este elemento, es por la conformación del sistema: brinzales, latizales, fustales y plantas herbáceas que extraen para su crecimiento (Acosta, 2008), al considerar a (Gerding \& Grez, 1996), es un elemento móvil y el requerimiento máximo de la especie en su etapa de mayor desarrollo foliar, absorben anualmente, de 30-55 kg de $\mathrm{N}$ frente a su aporte de $40 \mathrm{~kg} \mathrm{ha}^{-1}$ año-1 (Molinero, 2000).

Los valores de fósforo en los suelos franco arenosos y arena franca de la masa forestal en estudio fueron superiores a los reportados en suelos franco limosos de plantaciones en Cochabamba con valores: $5,6 \mathrm{mg} \mathrm{L}^{-1}$ a nivel del suelo y de $3,5 \mathrm{mg} \mathrm{L}^{-1}$ en el subsuelo (Flores, 2009). Los contenidos de fósforo son altos, particularidad que no se presenta en otros suelos, sumándose el ciclaje de nutrientes, en la etapa juvenil al requerir de 4-12 kg para el desarrollo de raíces (Gerding \& Grez, 1996) frente a su aporte por el desfronde con 1,4 $\mathrm{kg} \mathrm{ha}^{-1} \mathrm{año}^{-1}$ (Molinero, 2000). 
Flores (2009), al evaluar suelos de plantaciones de eucalipto con textura franco limosa encontró a nivel superficial 0,66 meq $100 \mathrm{~L}^{-1}$ y en subsuelo 0,45 meq $100 \mathrm{~L}^{-1}$ de potasio. Los resultados en el presente estudio muestran una mayor concentración, a medida que aumenta la profundidad del suelo. Sin embargo, una de las limitantes, es la compactación, la cual restringe su crecimiento, al absorber entre 6 y $30 \mathrm{~kg}_{\text {año }}{ }^{-1}$ de este elemento, cuyo aporte puede variar según el ciclaje de nutrientes (Gerding \& Grez, 1996).

Es bajo el contenido de boro en sitios con suelos de pH alcalino (Bell \& Dell, 2008), ocasiona pérdidas en el crecimiento y calidad de E. globulus (MAGAP, 2015) y (Lehto et al., 2010) al ser una de las especies más exigentes en B (Andrade et al., 1995), lo cual coincide con el valor obtenido en el suelo del área de estudio, asociado a suelos arenosos, bajo contenido de materia orgánica, sujetos a déficit hídricos, al requerir de este elemento para su rendimiento constituye una limitante (FAO, 2006).

Los valores de CIC en el área de estudio son bajos, lo cual es una limitante para retener una gran cantidad de cationes de los nutrientes Casanova et al., 2004) en concordancia con los resultados de textura franco arenosa que registra un valor de 3,2 Meq $100 \mathrm{~g}^{-1}$ en suelos reforestados con Eucalyptus robusta (Hernández et al., 2008), similar al 4,7 Meq $100 \mathrm{~g}^{-1}$ a nivel superficial y menor al valor de 14,9 Meq $100 \mathrm{~g}^{-1}$ reportados en suelos de E. grandis en Uruguay (Panario et al., 2007).

\subsection{Variables de establecimiento y manejo}

La aplicación de un plan de manejo para la plantación de E. globulus Labill; no coincide, con el ordenamiento, protección, conservación, restauración y aprovechamiento a ejecutar (Hernández, 2014); al no realizar un manejo adecuado, los resultados silviculturales son desalentadores como ocurrió en la masa forestal (Nalvarte, 2004).

\subsection{Variables dasométricas y estrategias de manejo.}

El incremento medio anual estimado en DAP de E. globulus Labill fue 2,55 veces inferior al valor promedio de 1,69 $\mathrm{cm}$ año ${ }^{-1}$ reportado en Biobío-Chile con una precipitación media anual de 1.150 mm a una altitud 320 msnm, con pendiente del terreno de $36,5 \%$ y 9 años de edad (Hubert et al., 2010).

La variable altura total promedio árbol ${ }^{-1}$ de la plantación en estudio resulto 2,64 veces inferior en relación con los $56 \mathrm{~m}$ en un rodal de similar edad (Mejía \& Moscoso, 2010). El incremento medio 
anual estimado menor, en referencia al valor de $1,47 \mathrm{~m}_{\text {año }}{ }^{-1}$ sobre un suelo poco apto de entre 13-16 años de edad (Moreno \& Igartúa, 2015).

El área basal promedio ha ${ }^{-1}$ proyectado, reportó un valor de 22,21 $\mathrm{m}^{2} \mathrm{ha}^{-1}$, ligeramente menor frente a 25,2 $\mathrm{m}^{2}$ ha $^{-1}$ en la región de Biobío (Hubert et al., 2010).

La densidad de cobertura en función del área basal, presentó valores entre 109,96 y 179,72 \% lo cual supera al $90 \%$, e indica aplicar raleo a los rodales 5, 6, y 7 (Alvarado, 2013).

El volumen total promedio árbol ${ }^{-1}$ del área de investigación es 2,34 veces menor al valor de $2,5 \mathrm{~m}^{3}$ árbol $^{-1}$ de un rodal de 40 años, cuyo incremento medio anual estimado resultó 1,53 veces menor a los 20,75 $\mathrm{m}^{3} \mathrm{ha}^{-1} \mathrm{año}^{-1}$ en Galicia (García \& Ruiz, 2003). Aunque al depender de la calidad de sitio, se han reportado en el Suroeste de España valores de 1,5 a $27 \mathrm{~m}^{3} \mathrm{ha}^{-1}$ año-1 y en Galicia entre 8 a $36 \mathrm{~m}^{3} \mathrm{ha}^{-1} \mathrm{año}^{-1}$ (Bravo \& Montero, 2008).

\section{Conclusiones y recomendaciones}

Los parámetros altitud, profundidad efectiva de raíces, contenido de nitrógeno y boro fueron determinantes en el bajo crecimiento y productividad de la especie $E$. globulus Labill en la hacienda Tunshi. Las asociaciones significativas de los parámetros que influyen en la productividad de la masa forestal son la altitud, materia orgánica, capacidad de intercambio catiónico y boro.

Si se toma en cuenta la pendiente estimada de 18 - $58 \%$, en el área desprovista de vegetación que se sitúa en la zona de conservación, en donde se propone realizar la reforestación, forestación con Acacia melanoxylon, Casuarina equisetifolia o Pinus canariensis y especies nativas en las épocas lluviosas, el diseño de plantación en tresbolillo con una equidistancia entre plantas de 3 metros.

El área de aprovechamiento forestal, rodales 5, 6 y 7 tiene una composición irregular en cuanto al número de árboles, área basal, volumen total, densidad en cobertura > $109 \%$, parámetros que sugieren realizar raleo a fin de evitar su pérdida por vuelco natural. En los rebrotes que se formarán luego de las cortas se deberá aplicar un manejo de renuevos, si es para madera de aserrío o laminada se dejaría entre 1-2 rebrotes tocón ${ }^{-1}$ a partir del año, coronamiento y fertilización. 


\section{Bibliografía}

Acosta, C. (2008). Evaluación de una fertilización en Eucalyptus globulus Labill aplicada en la etapa de máxima acumulación nutritiva (Trabajo de pregrado). Universidad Austral de Chile, Valdivia, Chile.

Afif Khouri, E., Canga Libano, E., Oliveira Prendes, J. A., Gorgoso Varela, J. J., \& Cámara Obregón, M. A. (2014). Crecimiento en volumen y estado nutricional de Eucalyptus globulus Labill y Pinus radiata D. Don en Asturias, España. Ciencias Forestales, 1(1).

Aguirre, Z., \& Aguirre, C. (2010). Las plantas vasculares como indicadores de la calidad y problemas de los ecosistemas. Ecología Forestal, I(1), 150.

Alvarado, M. (2013). Guía de silvicultura: Análisis y prescripción de comportamientos en planes de manejo con fines de silvicultura y manejo forestal. Obtenido de http://icf.gob.hn/wpcontent/uploads/2015/08/GUIA-DE-SILVICULTURA.pdf

Andrade, S., De Barros, N., Ferreira, R., Teixeira, J., \& Leal, P. (1995). Exigencia y distribución de boro en plantas de eucalipto. Bosque, 16, 53-59.

Bell, R. W., \& Dell, B. (2008). Micronutrients for sustainable food, feed, fibre and bioenergy production. Paris, France: International Fertilizer Industry Association.

Bravo, A., \& Montero, G. (2008). Descripción de los caracteres culturales de las principales especies forestales de España. Madrid, España: Instituto Nacional de Investigación y Tecnología Agraria y Alimentaria. INIA.

Bravo, C. (Febrero de 2014). Curso-taller de evaluación de la calidad física de los suelos. Riobamba: ESPOCH.

Casanova Pinto, M., Vera Elizondo, W., Luzio Leighton, W., \& Salazar Guerrero, O. (2004). Edafología. Obtenido de http://www.grn.cl/MANUAL\%20EDAFOLOGIA\%20_2004.pdf

Diaz Balteiro, L., Bertomeu, M., \& Giménez, J. (2008). Gestión óptima de Eucalyptus globulus en Galicia. Researchgate, 17.

Díaz Hernández, J., \& Sánchez Marañón, M. (1988-b). Aspectos edáficos del cultivo de eucalipto: Estado de conocimientos. Sevilla, España: Instituto Andaluz para la reforma Agraria, IARA. Junta de Andalucía.

Domingo-Santos, J. M., Corral, D. E., Redondo Salguero, F. R., Fernández de Villarán, S. R., \& Alesso, P. (2009). Influencia de la especie forestal en algunos parámetros edáficos en el SO español. Actas del V Congreso Forestal Español, Sociedad Española de Ciencias Forestales-Junta de Castilla y León. (Ávila, septiembre 2009). Ref electrónica: 5 CFE01093.

Ecuador forestal. (2010). Normas para el manejo sostenible de los bosques húmedos. Obtenido de http://ecuadorforestal.org/wp-content/uploads/2010/05/Normas-para-el-Manejo-ForestalSostenible-de-los-Bosques-H\%C3\%BAmedos.pdf 
FAO. (2009). Guía para la descripción de suelos. Obtenido de http://www.fao.org/3/a-a0541s.pdf

FAO. (2011). Diseños de muestreo de las Evaluaciones Forestales Nacionales. Obtenido de http://www.fao.org/fileadmin/user_upload/national_forest_assessment/images/PDFs/Spanis h/KR2_ES_4_.pdf

Flores, E. (2009). Efecto de las plantaciones de eucalipto (Eucalyptus globulus L.) sobre los suelos de comunidades asentadas en la red ferroviaria Cochabamba-Cliza. Vol. 4, № 2-3 . Cochabamba: Número especial: 1er Congreso Nacional de Sistemas Agroforestales.

García, O., \& Ruiz, F. (2003). A growth model for eucalyp in Galicia, Spain. Forest Ecology and Management, 173: 49-72.

Geldres, E., \& Schlatter, J. (2004). Crecimiento de las plantaciones de Eucalyptus globulus sobre suelos rojo arcillosos de la provincia de Osorno; Décima Región. Bosques, 25 (1), 95-101.

Gerding, V., \& Grez, R. (1996). Diagnóstico de deficiencias nutritivas en plantaciones forestales; Orientado a la aplicación de fertilizantes.Curso de Fertilización Forestal. 22. Valdivia, Chile: Universidad Austral de chile.

Grijalva et al. (2012). Situación de los recursos genéticos forestales en Ecuador. Informe país Ecuador. Quito.

Grupo Empresarial ENCE. (2009). La Gestión Forestal Sostenible y el Eucalipto. España: ENCE. Hernández, J. (2014). Curso Regional de Regulación, Manejo y Salud Forestal. Chihuahua.

Hernández, R., Ramirez, E., Castro, I., \& Cano , S. (2008). Cambios en indicadores de calidad de suelos de ladera reforestados con pinos (Pinus caribaea) y eucaliptos (Eucalyptus robusta). Agrociencia, 42 (3), 253-266.

Hubert, A., Iroumé, A., Mohr, C., \& Frene, C. (2010). Efecto de plantaciones de Pinus radiata y Eucalyptus globulus sobre el recurso agua en la Cordillera de la Costa de la región del Biobío, Chile. Bosque, 31(3), 219-230.

Instituto Nacional de Investigaciones Agropecuarias. INIAP. (2014). Resultados de Análisis de Suelos de la localidad Tunshi, provincia de Chimborazo. Quito.

Jaramillo, D. (2002). Introducción a la ciencia del suelo. Obtenido de http://www.bdigital.unal.edu.co/2242/1/70060838.2002.pdf

Kaplán, A., Labella, S., Rucks, L., \& Durán, A. (2011). Guía para la descripción e interpretación del perfil de suelo. Obtenido de http://www.cebra.com.uy/renare/media/Gu\%C3\%ADa-para-ladescripci\%C3\%B3n-e-intrepretaci\%C3\%B3n-del-perfil-del-suelo.pdf

Laboratorio de suelos de la Facultad de Recursos Naturales de la ESPOCH. (2014). Resultados de Análisis de Suelos de la localidad Tunshi, provincia de Chimborazo. Riobamba.

Lehto, T., Ruuholaa, T., \& Dellb, B. (2010). Boron in forest trees and forest trees and forest ecosystems. Forest Ecology and Management, 260, 2053-2069. doi: 10.1016/j.foreco.2010.09.028. 
Mejía, J., \& Moscoso, L. (2010). Efecto de las Plantaciones de Eucalipto (Eucalyptus globulus) y Pino (Pinus patula) en la Recuperación del Suelo y en la Regeneración Natural de la Cuenca Media del Río Paute. Cuenca, Ecuador: Universidad del Azuay, Cuenca, Ecuador. Ministerio del Ambiente del Ecuador. MAE. (2010). Aprovechamiento de los Recursos Forestales 2007-2009. Quito, Ecuador.

Ministerio de Agricultura, Ganadería, Acuacultura y Pesca. MAGAP. (2015). Programa de Incentivos para la Reforestación con Fines Comerciales. Guayaquil, Ecuador: MAGAP.

Molinero, J. (2000). Nitrógeno y fósforo en la materia orgánica particulada bentónica: influencia de la vegetación riparia, de las características del cauce y de la química del agua (Tesis Doctoral). Universidad del País Vasco.

Moreno, K., \& Igartúa, D. (2015). Eucalyptus globulus en el sudeste de la provincia de Buenos Aires: edades, procedencias y densidad de la madera. Revista de Investigaciones Agropecuarias, 41 (2), 143-149.

Nájera, J. A., \& Hernández, H. E. (2008). Relaciones morfométricas de un bosque coetáneo de la región del Salto, Durango. Ra Ximhai (4 (1)), 69-81.

Nalvarte, W. (2004). Silvicultura en la Amazonía Peruana.Diagnóstico de experiencias en la región de Ucayali y la provincia de Puerto Inca. CIFOR, IRENA, INIA, UN. Puculpalla, Perú.

Organización Mundial para la Agricultura y la Alimentación. FAO. (2006). Evaluación de Recursos Forestales mundiales. FRA 2005. Estudio FAO Montes. 147. Uruguay: FAO.

Palacios , W., \& Quiroz, H. (2012). Sondeo de percepciones sobre la rentabilidad del aprovechamiento de madera por pequeños propietarios. Proyecto USAID Costas y Bosques sostenibles. Quito, Ecuador.

Panario, D., Rabufetti, A., Taboada, M., Revel, J. C., \& Kaemmerer, M. (2007). Dinámica de la materia orgánica y de algunos parámetros fisicoquímicos, en la conversión de una pradera a cultivo forestal en la región de Piedras Coloradas-Algorta (Uruguay) (Titulo de doctorado). Institut National Polytechnique (INPT) de Toulouse, Francia.

Ramírez, L. (2012). Secuestro de carbono en plantaciones de eucalipto (Eucalyptus globulus Labill)- Cantón Pedro Moncayo, provincia de Pichincha, como una alternativa al desarrollo sustentable (Trabajo de pregrado). Universidad Técnica del Norte, Imbabura, Ecuador.

Sharma, M., Amateis, R., \& Burkhart, H. (2001). Top height definition and its effect on site index determination in thinned and unthinned loblolly pine plantations in: Forest Ecology and Management.

Sierra, R. (1999). Propuesta preliminar de un sistema de clasificación para el Ecuador Continental. Quito, Ecuador: Proyecto INEFAN/GEF-BIRF y EcoCiencia.

Soto, M. A. (2011). Izate, Asociación Medio Ambiental IZATE. Recuperado el 20 de febrero de 2014, de http://izate.blogspot.com/2014/02/problema de las plantaciones.html

Trujillo, E. (2013). Guía de reforestación. Bogotá, Colombia: El semillero. 